\title{
4G LTE Network Coverage Optimization Using Metaheuristic Approach
}

\author{
Zikrie Pramudia Alfarhisi \\ Department of Electrical \\ Engineering, Universitas \\ Brawijaya \\ Malang, Indonesia
}

\author{
Hadi Suyono \\ Department of Electrical \\ Engineering, Universitas \\ Brawijaya \\ Malang, Indonesia
}

\author{
Fakhriy Hario Partiansyah \\ Department of Electrical \\ Engineering, Universitas \\ Brawijaya \\ Malang, Indonesia
}

\begin{abstract}
The main focus of this paper is to optimize the coverage of each 4G LTE network cell within the service area. There are many algorithms can be implemented to determine the optimal 4G LTE coverage area including the deterministic and heuristic approaches. The deterministic approach could solve accurately the optimization problem but need more resources and time consuming to determine the convergence parameters. Therefore, the heuristic approaches were introduced to improve the deterministic approach drawback. The methods used are the Differential Evolution Algorithm (DEA) and Adaptive Mutation Genetic Algorithm (AMGA), which are categorized as metaheuristic approach. The DEA and AMGA algorithms have been widely used to solve combinatorial problems, including for solving the network optimizations. In the network optimization, coverage is strongly related to 2 objectives, which are reducing the black spot area and decreasing the overlapping coverage areas. Coverage overlap is a condition when some cell sites in an area overlap. It implies in the occurrence of hand off and an inefficient network management. This research aims to obtain an optimal 4G LTE network coverage and reduce the overlapping coverage areas based on effective e-Node B arrangements by using the DEA and AMGA algorithms. The simulations results showed that the DEA algorithm's coverage effectiveness was $23,4 \%$, and the AMGA Algorithm's was 16,32\%.
\end{abstract}

Keywords: Adaptive Mutation Genetic Algorithm (AMGA), Differential Evolution Algorithm (DEA), network coverage, optimization, 4G LTE

\section{INTRODUCTION}

Coverage area is one of the main factors to maintain the communication of mobile station users in 4G LTE network. The adequate coverage area is indicated by the network's ability to cover the service area with good signal quality [1]. The modelling of cell sites in the service area is strongly related to the optimum level of coverage. In optimizations, coverage has two main objectives, which are the decrement of blank spot area and the reduction of the overlapping coverage areas [2]. Coverage overlap refers to a condition where the cell sites in one area overlap, which often causes handoffs and a poor network management.

Differential Evolution Algorithm (DEA) and Adaptive Mutation Genetic Algorithm (AMGA) have been widely used to solve combinatorial problems, such as those which are found in network optimizations. Mendes et.al analyzed the use of Differential Evolution Algorithm (DEA) for the active network mapping which resulted in the optimization level of 0.02 [3]. In other research, Lestandy optimized the mesh network routing by using the Adaptive Mutation Genetic Algorithm (AMGA) which obtained the MC/GA result of 2,3\% [4]. Differential Evolution Algorithm (DEA) has unique characteristics in its mutation ratio and crossover ratio where the resulting probability will be adjusted to the inputs which contain the chromosome limitations [5]. Meanwhile, one of the benefits of the Adaptive Mutation Genetic Algorithm (AMGA) is it uses more complex mutation processes which are correlated with the desired fitness value [4].

The focus of this paper is to optimize the coverage of each cell of the 4G LTE network data in Mojokerto City, East Java, Indonesia, based on the e-Node B position coordinates. From the testing stage, optimization level of the network coverage in the service area will be obtained. The higher level of network coverage in the service area indicates the good performance of the methods used.

\section{CELL SITES CONCEPTS}

In cellular communications, information is interchanged between the Mobile Station (MS) and the Base Transceiver Station (BTS) via the radio signals. Each BTS can only communicate with the MSs within its coverage area. In other words, the radio signals deliveries are limited in a particular range of frequencies that several BTSs are needed to serve a wide area [6].

A BTS covers a certain area called a cell. The most common model of cells is some hexagonal with the same forms in the BTS's service area. Each cell provides some channels, that some of MSs can communicate with a BTS at the same time. A channel is usually defined according to time slots, the range of frequencies, encoding techniques, or a combination of TDMA, FDMA, and CDMA [7].

In cellular communication systems, as the amount of user traffic rises or the number of MSs increases, it is needed to add more channel's capacity. To add more channel's capacity, we can reduce the cell area's size (micro cell) or dynamically use the channels allocation and the re-use frequency. To plan the additional channel's capacity in a cellular system, we should consider the interference. There are two kinds of interference; those are the co-channel interference and the adjacent channel interference.

A group of adjacent cells which uses the whole frequency allocation is called a cluster size or a re-use frequency factor. According to the variation of their size and coverage, cells are categorized to femto, pico, micro, macro, and mega cells. Femto cells are usually used for connecting personal devices such as laptops. Pico cells usually cover a room or a part of a room in a building. The micro cells' coverage area is within 
an urban area and the macro cells' is within a sub-urban area. Mega cells are usually used in satellite communications, as they cover an area of up to hundreds of kilometers.

\section{RADIO PROPAGATION}

Knowledges about radio propagation's characteristics are required to plan a cellular communication system design. Different from the regular communications, the environment profiles of cellular communication systems are hard to predict. Radio propagation is determined by the area's profiles, moving objects, radio frequency properties, the MS's speed, and interference sources.

The signal propagation mechanisms between a transmitter and a receiver vary depending on the area's profiles around the cellular communication environment. This causes the signals received by MS to fluctuate. Signal fluctuations can occur in three mechanisms: reflection, diffraction, and scatter.

\section{METAHEURISTIC ALGORITHMS}

The metaheuristic algorithms emerged as a new approach to solve the limitations of heuristic algorithms. Metaheuristic algorithms are inspired by events in nature, or better known as the nature inspired algorithms. This approach is simpler and easier to implement into the computer programming languages so it can provide faster solutions than the heuristic algorithms [8]

Some methods of the metaheuristic approach which can be used for solving various combinatorial optimization problems are Genetic Algorithm (GA), Cross Entropy (CE), Particle Swarm Optimization (PSO) and Ant Colony Optimization (ACO).

\section{DIFFERENTIAL EVOLUTION ALGORITHM (DEA)}

Differential Evolution Algorithm (DEA) is included in the family of Evolutionary Algorithms (EA), which are evolutionary population-based algorithms. The algorithms' principles and philosophy simulate the biological evolution behaviors. The DEA was introduced by Storn and Price in 1995. DEA differs from the other type of Evolutionary Algorithms in the way to determine the distance and direction of the population's/solution's searching process.

According to Storn and Price, optimization techniques should in general meet 3 conditions [5]. First, the method must find a global optimum, despite the system's first parameters' values. Second, the convergence must be fast. Third, the program must have minimum limits of control parameters' values. Those conditions underlie the emergence of Differential Evolution Algorithms (DEA). DEA refines other types of Evolutionary Algorithms (EA) with a simple optimization strategy for a prompt optimization process (a shorter execution time with less literacy to find the global optimal solution)

\section{ADAPTIVE MUTATION GENETIC ALGORITHM (DEA)}

Genetic Algorithm (GA) is a method which is included in Evolutionary Algorithms (EA) family. In common, Evolutionary Algorithms (EA) imitates the natural process of evolution, where the main concept is the superior individuals will survive, while the inferior ones will become extinct [9]. The superiority of the individuals is measured through a mathematical function called the fitness function. The term of fitness in GA refers to the feasibility of a solution to the problem.

In GA, the mutation probability (Pm) value is constant, and this results in the lack of the optimization's efficiency. The Adaptive Mutation Genetic Algorithm (AMGA) uses adaptive mutations to prevent premature convergence [4].

\section{RESEARCH METHODOLOGY}

This research discusses the optimization of the 4G LTE's network coverage by using the metaheuristic approach. The testing stage was conducted through simulations in Python programming language by using the open-source Python notebook of Google Collaboratory.

The type of data used in this research was secondary data which were collected from books and research related to the topic. Secondary data used were the service area's geographical condition, cell's distribution of the cellular network, and the population density data. The functions of those data are:

1. The service area's geographical condition data were collected from the map of the service area. The data included special characteristics of the area, and those used to underlie the mappings of the cellular network.

2. Cells' distribution data included the position coordinates of communication provider's Base Transceiver Stations (BTSs) or e-Node Bs in the service area. From the preliminary data, we obtained the existing condition of the cellular communication's signal coverage. In this research, the cells' distribution would be repositioned according to the optimization results.

The cells' distribution data of Mojokerto City, East Java, Indonesia as well as the mutation and crossover ratio were used as inputs of the optimization models. The optimizations were conducted by using the Differential Evolution Algorithm (DEA) and the Adaptive Mutation Genetic Algorithm (AMGA). The inputs, processes, and outputs flow of this research is shown in the Figure. 1.

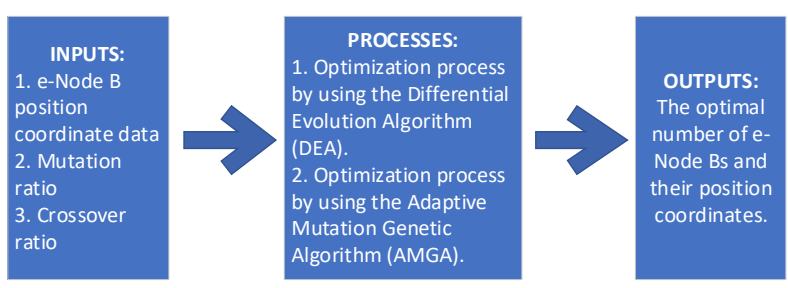

Figure. 1 Inputs, processes, and outputs flow diagram of the research

In general, the optimization processes in this research were started by collecting the input data, as follows:

1. The data of e-Node Bs' position coordinates in the service area. Those data contained the longitudes and latitudes of existing e-Node Bs which were the descriptions of the special data. Those position coordinates data were used in both Differential Evolution Algorithm (DEA) and Adaptive Mutation Genetic algorithm (AMGA) models.

2. Mutation ratio. This included the number of genes that should be used in the mutation steps in the Adaptive Mutation Genetic Algorithm (AMGA) optimization. On 
implementation, Differential Evolution Algorithm (DEA) and Adaptive Mutation Genetic Algorithm (AMGA) have different mutation characteristics.

3. Crossover ratio. This was the ratio of offspring which are produced in crossover stage in every generation.

After defining the inputs, the optimization processes were conducted by using the Differential Evolution Algorithm (DEA) and Adaptive Mutation Genetic Algorithm (AMGA). From the optimization processes, we obtained the optimal numbers of e-Node B points which should be implemented in the service area as outputs. Those numbers described the effective levels of the coverage necessity within an area which were tabulated in a coordinate points table. From each algorithm which was implemented in this research, we obtained different outputs which would be compared and analyzed. Optimization parameters used in the simulations in this research for the DEA and AMGA are shown in the Table I and Table II, respectively.

Table 1. Optimization parameters of the Differential Evolution Algorithm (DEA)

\begin{tabular}{|l|c|}
\hline \multicolumn{1}{|c|}{ Parameter } & Parameter's value \\
\hline Population's dimension & 98 \\
\hline Population's size & 10 \\
\hline Preliminary population's size & 50 \\
\hline Maximum iteration & 300 \\
\hline Mutation Ratio (F) & 0.7 \\
\hline Crossover Ratio (CR) & 0.6 \\
\hline
\end{tabular}

Table 2. Optimization parameters of the Adaptive Mutation Genetic Algorithm (AMGA)

\begin{tabular}{|l|c|}
\hline \multicolumn{1}{|c|}{ Parameter } & Parameter's value \\
\hline Population's dimension & 98 \\
\hline Population's size & 10 \\
\hline Preliminary population's size & 50 \\
\hline Maximum iteration & 300 \\
\hline Preliminary mutation ratios & 0.1 \\
\hline
\end{tabular}

\section{RESULTS AND DISCUSSIONS}

From the simulations, we obtained the number and the position coordinates of the e-Node Bs should be activated in the service area. The testing results of the Differential Evolution Algorithm (DEA) model is shown in the Figure. 2.

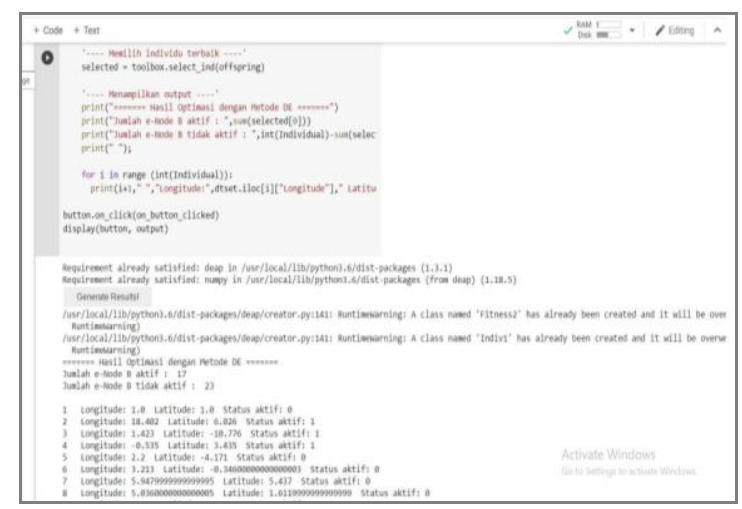

Figure. 2 The testing results of Differential Evolution (DE) method in Google Collaboratory

Fig 2. shows the number of e-Node Bs which are activated based on the optimization result. The Differential Evolution Algorithm (DEA) model generated 23 inactivated e-Node Bs and 17 activated e-Node Bs of 98 existing e-Node Bs.
Moreover, we also get the activation status of each e-Node B based on the optimization results.

On the other hand, the Adaptive Mutation Genetic Algorithm (AMGA) model generated 16 inactivated e-Node Bs and 24 activated e-Node B of existing 98 e-Node Bs. We also obtained the position coordinates and the activation status of each e-Node B in the service area. The testing results of the Adaptive Mutation Genetic Algorithm (AMGA) model can be seen in Fig 3.

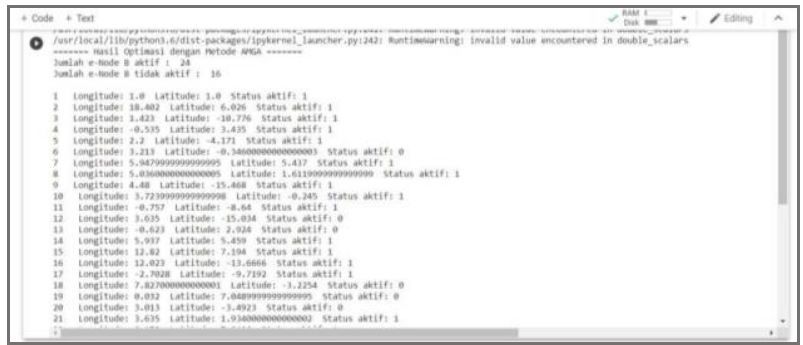

Figure. 3 The testing results of Differential Evolution (DE) method in Google Collaboratory

In existing condition, there are 98 e-Node B points to meet the coverage necessity of a $36.56 \mathrm{~km} 2$ area with 165.362 population. From the testing results we can see that the necessity of network coverage in the service area can be meet with less e-Node B points.

From the testing stage, the Differential Evolution Algorithm (DEA) model generated 23 inactivated e-Node B points, those are e-Node B number $2,8,20,21,34,38,39,41,47,54,59$, $60,65,69,70,74,80,81,83,86,87,90$, dan 94 . This means there were overlapping coverage areas between some cell sites in the existing condition. Moreover, there were 17 activated eNode B points to satisfy the necessities of network coverage in some areas that had poor coverage. Differential Evolution Algorithm (DEA) model resulted in $23.4 \%$ effectivity rate compared with the existing condition with evenly spread coverage.

On the other hand, the Adaptive Mutation Genetic Algorithm (AMGA) model resulted in 16 inactivated e-Node B points, those are e-Node B number 4, 8, 9, 21, 24, 34, 38, 39, 41, 47, $58,67,83,90$ dan 94 . Then, there were 24 activated e-Node B points to meet the coverage necessities of some areas that had poor coverage. According to the testing results, the Adaptive Mutation Genetic Algorithm (AMGA) model got 16,8\% effectivity rate compared with the existing condition. The comparison between the existing condition and the optimization result with the metaheuristic approach is shown in Fig 4 and Fig 5.

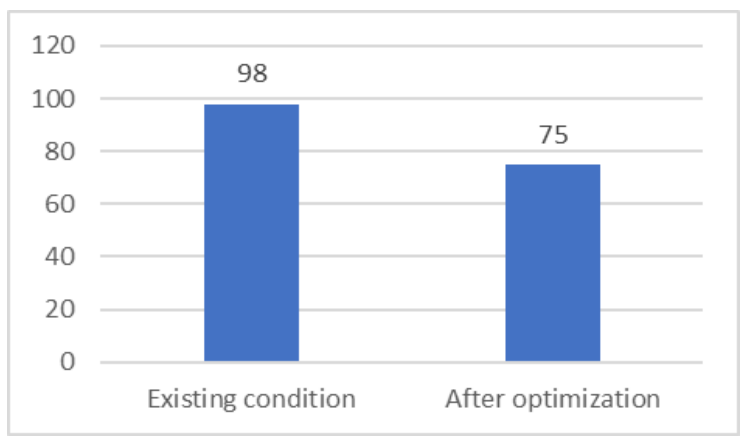

Figure. 4 Optimization's result of the Differential Evolution Algorithm (DEA) 


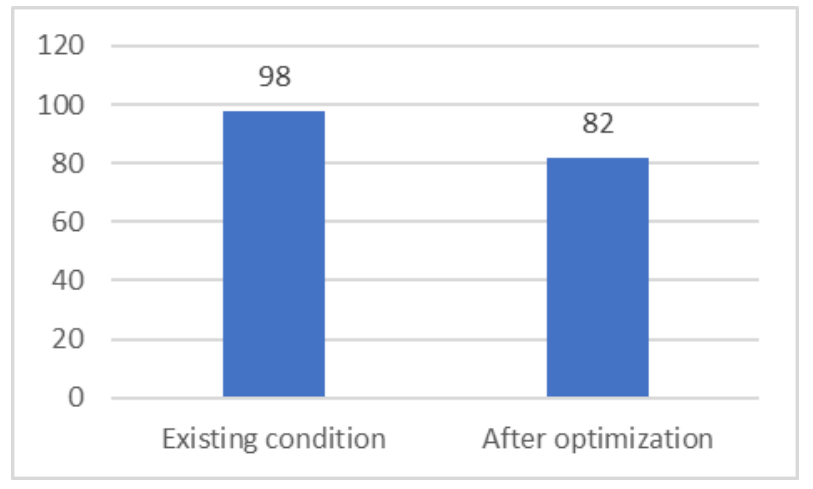

Figure. 5 Optimization's result of the Adaptive Mutation Genetic Algorithm (AMGA)

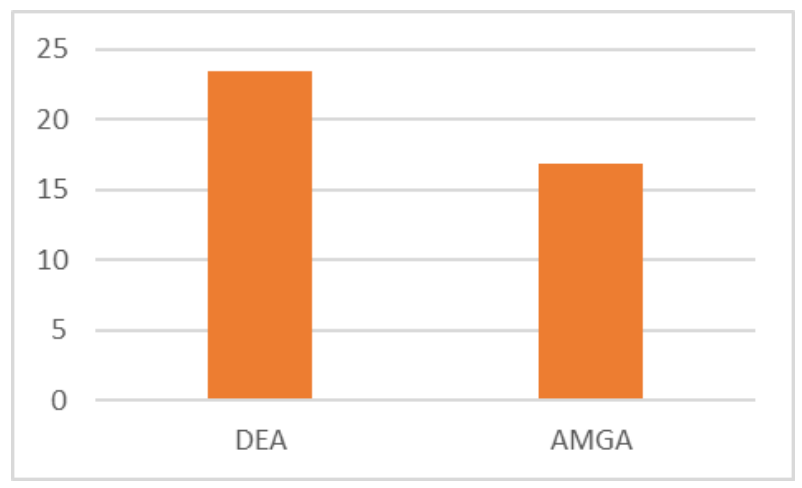

Figure. 6 Comparison between the optimization result of DEA and AMGA

According to the testing results, the effectivity rate of the Differential Evolution Algorithm (DEA) model is $23,4 \%$ dan Adaptive Mutation Genetic Algorithm (AMGA)'s is 16,8\%. We can conclude that both metaheuristic methods have better results than the existing condition.

\section{CONCLUSION}

This research focuses on optimizing the 4G LTE's network coverage by using metaheuristic approach. The Differential Evolution Algorithm (DEA) and the Adaptive Mutation Genetic Algorithm (AMGA) have been proposed to determine the optimal coverage of the 4G LTE network. The Differential Evolution Algorithm (DEA) and the Adaptive Mutation Genetic Algorithm (AMGA) could be implemented to solve the network coverage optimization problem, especially for reducing overlapping coverage areas. The Differential Evolution Algorithm (DEA) model resulted in $23,4 \%$ of coverage effectivity rate compared with the existing condition. The Adaptive Mutation Genetic Algorithm (AMGA) model resulted in $16,32 \%$ of coverage effectivity rate. The future research can be conducted by implementing non-heuristic methods to solve the network coverage optimization problem of 4G LTE network. Moreover, we can add the other QoS parameter, such as traffic capacity.

\section{REFERENCES}

[1] Joonas Säe, Jukka Lempiäinen, "Maintaining Mobile Network Coverage Availability in Disturbance Scenarios", Mobile Information Systems, vol. 2016, Article ID 4816325, 10 pages, 2016 https://doi.org/10.1155/2016/4816325

[2] J. Wiley, Small Cell Optimization, Hoboken, NJ, USA: Wiley Library, 2015.

[3] S. P. Mendes, J. A. Gomez Pulido, M. A. Vega Rodriguez, M. D. Jaraiz Simon and J. M. Sanchez Perez, "A Differential Evolution Based Algorithm to Optimize the Radio Network Design Problem," 2006 Second IEEE International Conference on e-Science and Grid Computing (e-Science'06), Amsterdam, The Netherlands, 2006, pp. 119-119, doi: 10.1109/ESCIENCE.2006.261052

[4] Lestandy, M., Pramono S.H,, Aswin M., "Routing Optimization on Metropolitan Mesh Network Using Adaptive Mutation Genetic Algorithm" (Indonesian Version), November 2017, Jurnal Nasional Teknik Elektro dan Teknologi Informasi (JNTETI) 6(4), DOI: 10.22146/jnteti.v6i4.355

[5] D. Chaudhary, A. K. Tailor, V. P. Sharma and S. Chaturvedi, "HyGADE: Hybrid of Genetic Algorithm and Differential Evolution Algorithm," 2019 10th International Conference on Computing, Communication and Networking Technologies (ICCCNT), Kanpur, India, 2019, pp. 1-4, doi 10.1109/ICCCNT45670.2019.8944822.

[6] B. Zhuang, D. Guo and M. L. Honig, "Energy-Efficient Cell Activation, User Association, and Spectrum Allocation in Heterogeneous Networks," in IEEE Journal on Selected Areas in Communications, vol. 34, no. 4, pp. 823-831, April 2016, doi: 10.1109/JSAC.2016.2544478.

[7] Allen W. Scott; Rex Frobenius, "Multiple Access Techniques: FDMA, TDMA, AND CDMA," in RF Measurements for Cellular Phones and Wireless Data Systems , IEEE, 2008, pp.413-429, doi: 10.1002/9780470378014.ch30.

[8] X.-S. Yang, "Nature-Inspired Metaheuristic Algorithms: Success and New Challenges," J Comput. Eng. Inf. Technol., vol. 1, no. 1, pp. 1-3, 2012.

[9] A. P. Engelbrecht, Fundamentals of Computational Swarm Intelligence, Hoboken, NJ, USA: John Wiley \& Sons, 2006. 